\title{
现代汉语总结类话语标记浅析 Discourse Markers of Summary in Modern Chinese
}

\author{
曹秀玲* \\ 刘流 \\ 上海师范大学对外汉语学院 \\ 上海 中国, 200234
}

xlcao@shnu.edu.cn

342368761@qq.com

Shanghai Normal University

International College of Chinese Studies Shanghai, China 200234
Cao Xiuling

Liu Liu
总结类话语标记是人类语言共有的元话语 成分之一。现代汉语总结类话语标记包括 “总 之”、“一句话”、“概括起来讲”、“总的 来看”等众多成员，根据构成成分及其结构关 系特点可以归纳为以 “话/言” 和 “说/讲/ 看” 为核心成分的名词和动词两个系列。总结 类话语标记分布在前后语段之间, 构建的语篇 模式为 “ $a, x, b$, 在 “要点概括” 的总体语 义功能之下表达概括、推论、补充、转折、让 步等多种下位语义关系。总结类话语标记的语 篇组织功能和人际互动功能是其概念表达功能 语法化的结果, 在这一过程中从基本话语发展 为元话语。 


\section{1. 引言}

传统意义上的语法包括词法和句法两个部分, 语法分析时一般将无法划分 为主、谓、宾和定、状、补等六大句子成分的语言单位被称为 “插入语” 或 “独 立成分”。随着上世纪六、七十年代篇章语言学的兴起, 语法研究视域逐渐从 句法扩展到章法，越来越关注句际之间的关系以及不同小句如何整合为语篇。

篇章之所以成为篇章, 是因为具有篇章特征（texture）。de Beaugrande 和 Dressler(1981) 提出了篇章具有七个要素, 即衔接、连贯、 意图性、可接受性、信息性、情景性、互文性。尽管目前人们尚不确知并清晰 阐明篇章特征究竟有哪些, 但衔接和连贯无疑是其中一对最基本的概念。对于 二者的关系，韩礼德和哈森(1976）提出, 衔接是 “建造连贯大厦的基础”。 学界的共识是, 语义连贯是篇章的核心特征之一, 而要实现语义连贯必然需要 采取一定的衔接手段。

就目前所知, 将多个小句整合为一个语篇并确保语义连贯而采取的形式手 段可以归结为 “加” 和 “减” 两种做法。前者是指通过添加连接成分, 实现语 句之间的有形衔接并外显语句之间的语义关系; 后者通过句法降级减弱某些小 句的独立性从而增强其对语篇的依附性，实现小句的无形衔接。本文以总结类 话语标记为例展示小句与语篇之间的依存关系以及篇章连接成分在语篇构建 过程中所 “扮演” 的重要角色。

\section{2. 总结类话语标记的界定}

廖秋忠（1986）勾勒了汉语篇章连接成分的总体框架，将其归纳为顺接、 逆接和转接等三种。其中总结类篇章连接成分属于顺接的一种，包括 “总（而 言）之、总起来说/讲、总括起来说、一句话、一言以蔽之、概括起来说、总 的看 (来) ”等多种表现形式。在实际语言表达中, 上述连接成分还有很多变 体形式, 如 “概括/总括起来讲, 总体来说/讲、总的看/说来、总的来说/讲” 等。这类成分连接前后多个小句并标明其间的语义关系，调控言语表达进程。 
根据话语分析理论, 每一次言语行为都包括基本话语和元话语两个层面, 前者是指那些具有指称和命题信息的话语; 后者是指 “关于基本话语的话语”, 即对命题态度、语篇意义和人际意义进行陈述的话语。总结类篇章连接成分是 元话语的一个小类, 本文着眼于基本话语和元话语之间的区别和联系, 称之为 “总结类话语标记”。1

\section{3. 总结类话语标记的成员及其构建的语篇模式}

\section{1 总结类话语标记的成员}

如前所示, 总结类话语标记的成员众多,构造方式也不尽相同。刘流(2015) 根据总结类话语标记构成成分, 将其归结为（1） “看” 类、（2） “说/讲/ 述” 类、（3）“一”类、（4）“总” 类和（5）“归” 类（如 “归根结底”） 等五个小类。由于总结类话语标记由多个构成成分组成, 上述分类之间难免有 交叉。

如果兼顾构成成分及其结构关系特点, 总结类话语标记可以归结为以名词 “话/言” 和动词 “说/看/讲 (/言) / 述” 为中心成分的两大系列。其中前一系 列主要包括 “一句话” 和 “一言以蔽之”，后一系列成员数目更多，构成也相 对比较复杂： “总的/总体” 直接修饰动词的用例相对较少, 主要采用 “总的/ 总体 $V$ 来” 或 “总的/总体来 $V$ ” 形式，也有 “总括/概括起来” 与 “说/看/讲” 组合的形式, 另有 “综上所述” 和 “总 (而言) 之” 等书面色彩较强而且构成 更为复杂的总结类话语标记形式。

由于话语标记的多源性与非排他性（曹秀玲、辛慧 2012），上述众多总 结类话语标记实际上是语义近乎等值而语体色彩略有差异、使用频度有高低之 别的变体形式，如 “一言以蔽之”、“综上所述” 较少出现在口语当中，而“一 句话” 和 “总的 (来) /说 (来) ”极少出现在书面语中, 其他总结类话语标 记的语体分布相对比较自由。据刘流 (2015) 对 CCL 语料库 ${ }^{2}$ 的穷尽统计, 使 用频度 500 次以上的总结类话语标记由高到低依次为: 总之（8898）、总的来 说（1073）、总而言之（1057）、归根结底（679）、综上所述（562）。

基本话语和元话语之间处于连续统状态, 很多元话语是基本话语虚化的结果, 总结类话 语标记也不例外，本文只关注其共时层面作为话语标记的用法。

本文语料如无特别说明, 均出自北京大学中国语言学中心语料库, 谨致谢忱。 


\section{2 总结类话语标记构建的语篇模式}

3.2.1 总结类话语标记作为元话语, 表现出较强的句法独立性, 不与相 邻的成分构成更大的语法单位, 甚至出现与否不影响所在语句的合法性。例如:

（1）这时我对蒋介石也十分不痛快：第一，自罗、史到后，有关作战方面， 蒋对我无直接指示; 第二, 我二十一日陈述集中主力于梅苗、棠吉间作持久战 的意见，蒋始终未复，不知他的意图; 第三，我认为蒋二十四日 “手启” 电是 未了解棠吉二百师的战绩, 决心变得过早, 给史、罗钻了空子, 转发命令来威 胁我; 第四，到这时已将远征军弄得一塌糊涂，很难挽回危局。总之，我认 为蒋介石太相信史、罗已将战局搞坏，再向他们说话也就无用。（《中国远征 军入缅对日作战述略》)

（2）任您怎么说，这几年有些专门玩文化的行业硬是玩不下去啦一一电影 院里除了情侣外, 别的人不多; 令老百姓仰慕的京剧团也多数解散了; 书店里 更是买书的和卖书的差不多; 连那些一向耻于谈钱的作家们也争先恐后地下海 了。一句话, 文化这碗饭不好吃了。（市场报1994 年 B)

上述例句中，“总之”、“一句话” 前面都是完整的表述形式，在书面中 表现为以句号形式结句，而逗号将 “总之、一句话” 与后面表述隔开。也就是 说，“总之、一句话” 处于前后表述之间，“桥接” 分述和总结部分，体现人 类语言联系项居中的总体趋势。将上述例文中的 “总之、一句话” 删略未尝不 可, 但是前后表述之间的语义关系无法得以外显。当然, 在口语表达中, 语气 词、停顿等非语言形式可以弥补语言形式缺失带来的语篇衔接和理解困难。

在口语等即兴表达中, 有时总结类话语标记也有作为追加形式出现在句尾 的情况。我们知道, 言说者不会刻意追加一个不必要的成分, 言说者这种下意 识的言语表达形式也可佐证其在语篇构建过程中的重要作用。

3. 2.2 总结类话语标记关联小句、句子和语段等多种语言单位。具体用 例如:

（3）给我看病的医生，即禁止我作任何旅行的那位，劝父母不要让我去看 戏，说我回来以后会生病的，而且可能病得很久，总之，我的痛苦将大于乐 趣。(马塞尔・普鲁斯特《追忆似水年华》)

（4）“教育儿子是父亲的义务吧?”“一般而言，是的。”可是，那并不 意味着凡事都要模仿父亲, 走和父亲一样的路。总之, 如果还有其它爱好的 话，立志当个学者或艺术家都好，我会全力支持的。（田中芳树《银河英雄传 说》) 
（5）怎样才能留下深刻的第一印象呢? 首先要表现出活力。眼睛看着主考 官，但又不死死盯着 $>^{3}$

其次要表现出主动，首先主动自我介绍一下，……然后 $\diamond$

最后要礼貌适度。开始时要问候主考官，结束时要谢谢他们 $\diamond$

总之, 面试时要主动一些, 活跃一些, 仅仅中规中矩, 很难脱颖而出。 (百度网)

上面三个语例中的总结类话语标记依次出现在小句、句际和段落之间，连 贯起前后语言片段。如果将总结类话语标记前后的语言片段分别标记为 $\mathrm{a}$ 和 $\mathrm{b}$, 而总结类话语标记为 $X$, 那么语篇模式可以码化为: $a, X, b$ 。单从语符系列 的长度来看, 一般 $\mathrm{b}$ 远远小于 $\mathrm{a}$, 而从语义重心来看, $\mathrm{b}$ 往往是在 $\mathrm{a}$ 分述基础 上高度凝练的表达形式, 因此往往具有以简驭繁、画龙点睛之效。从这种意义 上说，b段重于 $\mathrm{a}$ 段。（例见前文（1）、（2））

\section{4. 总结类话语标记表达的语义关系}

顾名思义, 总结类话语标记表明其后语段 $b$ 是对前面语段 $\mathrm{a}$ 的总结和概括。 廖秋忠 (1986) 指出, “总结连接成分就是把前面已说过的话加以归纳, 用简 单的一句话或几句话把要点概括出来。” 语言事实表明, 在 “归纳、总结”、 “要点概括” 等总体框架之下, 总结类话语标记所连接的前后语段之间存在多 种下位语义关系。刘菲露（2008）提出，“总之” 的语义关系有推论、强化、 目的、解说、承接、转折和假设七种。李绍群 (2013) 提出, “一句话” 的语 义关系有并列或递进、因果、目的、补充。根据笔者的考察，总结类话语标记 所表达的前后语段之间的下位语义关系可以概括为: 总分关系、推论关系、补 充关系、转折关系和递进关系。这些下位语义关系往往通过一定的语言形式加 以标识, 下面分别举例说明。

（6）近年来，上海对市区儿童进行智力测验，结果也表明，在 12 项测验 中，我国儿童 7 项超过美国儿童，3项与美国儿童相似，2 项低于美国儿童。 总的说, 中国人是智力优秀者。（曾鹏飞《技术贸易实务》）

（7）“这意思是事情就这样了结了, 福尔摩斯回答道。”而且, 总的来看, 这可能是最好的结局。(阿瑟·柯南道尔《福尔摩斯探案集》)

（8）〉综上所述，俄国近年来兴起的社会民主主义运动具有先天不足、路

由于例文较长, 为节省篇幅, 本文用 “ $\diamond ”$ 表示引文中省略的部分以区别于原文的省略 用法, 下同。 
途坎坷、形象不明、组织多变的特点。这表明它的发展水平与东欧各国相比 （更不用说与西欧或北欧相比）是较为低级的。（网络语料 C000023）

（9）如果他们靠近他，或是做出什么突然的动作，他会猛然退缩; 而且, 他也刻意避开他们的精灵斗篷。不过，总体来说，他还是非常的友善、费尽 心力想要讨好人，让人看了很不忍心。（托尔金《魔戒》）

（10）她接着说：“做了整容手术后，增强了自信感，竞技水平也得到了 提高。一句话, 能够自信地对待一切。”（百度）

上面例（6）通过中美儿童测试成绩对照, 得出 “中国人是智力优秀者” 的结论; 例（7）语段 b 的 “可能” 突显前后语段之间不确定的推理关系; 例 (8)在叙述走过十年风雨历程的俄罗斯联邦共产党所进行的左派运动的基础上 补充其特点; 例（9）语段 a 陈述 “他们” 对人类的身朵避，而话锋一转说 “他 们”也有讨好人的一面; 例（10）语段陈述整容手术带来的积极作用，不但自 信心增强, 竞技水平提高, 语段 $\mathrm{b}$ 在此基础上推进一步, 能够自信地对待一切。

\section{5. 总结类话语标记的元话语功能}

韩礼德 (1985) 指出语言具有三种基本功能, 即概念功能、语篇功能和人 际功能。总结类话语标记作为元话语成分, 主要具有语篇功能和人际功能。然 而, 其某些变体形式在共时层面仍保留一定的概念功能。请看下面的例句:

（11）水泥质量总体来说是好的，逐年在提高，但尚有 $5 \%$ 左右的样品不合 格, 对此我们不能掉以轻心。（1994 年报刊精选）

（12）上钢三厂的薪点工资制总的看还处于探索前进阶段，如管理人员和 科技人员的量化考核，如何排除人为因素问题; 一些职工因为所处位置不同， 对某些薪点有不同意见。（1994 年报刊精选）

（13）中国专制官僚政治上的帝王绝对支配权归根结底是建立在全社会基 本生产手段一土地的全面控制上, 是建立在由那种基本生产手段的控制所勒 取的农业剩余劳动或其劳动生产物的占有上。（1994 年报刊精选）

上面例句中的 “总体来说/总的看” 和 “归根结底” 在句中作状语, 分别 表示从全局、整体而非局部、部分和从本质而非表象方面加以评判。

作为元话语的总结类话语标记只能出现在前后语段之间, 实现语篇和人际 功能, 具体表现为语篇衔接、信息传递和人际互动三个方面。 


\section{1 语篇衔接功能}

总结类话语标记昭示前后语段之间的层次关系，使得前后语段语义连贯、 自然、显豁，并将前后语段整合为一个有机的语篇片段。例如:

（14）通过这件小事，使我切身感到：《作家文摘》的门槛虽高，但并不 难进; 名家作品虽多，可并不冷眼相待小人物的稿件，一句话，在稿件选用 上一视同仁! (崔仲岐《编读往来》)

（15）接你第二封信，似乎要回信，说破你的悲哀，似乎不必回信，恐怕 增你的反感。总之，我不想回信，等到九月回京也不想写信，而且无论到何 时都不想写信, 可以说：是我再不想给你的信。（白薇《情书》）

上述两例中的 “一句话”、“总之” 不充当任何句法成分, 看似可以删略, 但从语篇角度看, 删略之后, 前后语段语义关系改变甚至不连贯: 例 (14) 后 续句变为前面语句的延续，而非对前文的概括总结；例（15）前后语段将 “各 自为战” ，不是一个有机的整体。由此可见，虽然汉语语法具有一定的意合特 点，但合语法的两个语段简单并置并不天然构成合格的语篇。

廖秋忠在《篇章中的管界问题》一文将 “篇章管界” 定义为某个管界词语, 如动词、各种修饰语等所支配、修饰或统领的范围, 若统领范围跨越句子边界, 就是篇章管界。以上用例表明, 总结类话语标记是语篇的 “粘合剂” ，它连接 前后语段，但在语义上统领其后语段并使之成为语篇信息焦点。

\section{2 信息传递功能}

根据关联理论，人们在会话中总是希望以最小的努力获得最佳的语境效 果, 而话语标记的使用恰好能最大限度地减少听者对话语处理而付出的努力。 de Beaugrande 和 Dressler (1981：72) 将篇章信息分为三个层次:一级信息, 信息值不高, 缺乏可读性; 二级信息, 信息值高, 需要付出一定的努力; 三级 信息, 信息值需要很强的处理能力才能达到。而言说者为了使会话得以继续进 行, 需要平衡各层级信息值, 最重要的方式就是信息升级和降级。

对言说者来说, 总结类话语标记能为话语活动提供话语线索与标记, 降低 理解难度，有助于听读者 “按图索䩀” ，根据这一标记的提示来对话语进行分 析与处理，从而达到正确理解话语、有效交流的目的，是一种信息升级的形式。 例如:

（16）杜大叔跟这个从城里来的青年人一块生活了一天一夜，用一张无形 的卷子考过他, 对他的人品, 做了一次深刻的鉴定。从这里边, 杜大叔心里产 生一种说不出来的喜悦, 一句话, 已经很喜欢这个年轻人了。（浩然《夏青 苗求师》) 
（17）民间众说纷纭，总而言之说坐月子就是要在床上睡三十天到四十五 天。（池莉《太阳出世》）

上面例 (16) 和 (17) 中, 言说者为引导听读者准确地理解话语, 分别使 用总结类话语标记 “一句话” 和 “总而言之” 概括总结前文内容, 将前文四个 小句的陈述和民间各种说法总结为一句话, 从而突显会话重点和关键信息。

\section{3 人际互动功能}

语言表达具有主观性的一面，沈家煊（2001）提出 “主观性是指语言的这 样一种特性, 即在话语中多多少少总是含有说话人 “自我” 的表现成分, 也就 是说, 说话人在说出一段话的同时表明自己对这段话的立场、态度和感情, 从 而话语中留下自我的印记。”与此同时, 言语交际也表现出交互主观性的一面: 口语交谈固然是听说双方的互动交谈，而书面语言也往往采用 “拟对话” 的形 式营造生动形象的交际场景（李宇明 1996，曹秀玲、王清华 2015），从而更 好地实现作者和读者之间的互动。从人类语言交际功能的特质看, 自然语言中 的很多表达方式更多地在于协调说话人/作者与听话人/读者就某一命题的认 知立场。这是因为, 语言交际中作为言语主体的参与者不仅能够意识到同样作 为言语主体的交际对方的存在，而且表达出对交际对方认知 “自我” 的关注， 所以交互主观性强调信息的共有性。例如:

（18）做政治需要迷叶，不然便见不到皇帝。作军官需要迷树，它是军饷。 作诗必定要迷叶, 它能使人白天作梦。总之, 迷叶是万能的, 有了它便可以 横行一世。（老舍《猫城记》)

（19）他们首先选择的，正是 “走西口”。口外，为数不小的驻防军队需 要粮秝, 大片的土地需要有人耕种; 耕种者、军人和蒙古游牧部落需要大量的 生活用品，期待着一支民间贸易队伍; 塞北的毛皮、呢线原料是内地贵贵之家 的必需品, 为商贩们留出了很多机会; 商事往返的频繁又呼唤着大量旅舍、客 店、饭庄的出现……总而言之, 只要敢于走出去悉心寻求、刻苦努力, 口外 确实能创造出一块生气勃勃的生命空间。(余秋雨《抱愧山西》)

上面两段例文中，总结类话语标记前、后语段语符列长度严重 “失衡”。 为便于听读者理解语段 $\mathrm{a}$ 的要点, 说写者在前文具体铺排详叙的基础上以总结 类话语标记为提示语, 简明扼要地加以概括, 凸显会话重点, 帮助对方获取并 理解关键信息, 从而达成交际目的。

\section{6. 结语}

总结类话语标记是人类语言的共性表现之一, 该类话语标记的语篇组织功 
能和人际互动功能是概念表达功能虚化的结果, 而它在语篇中所实现的语篇组 织功能和人际互动功能归根结底是语言作为人类交际工具这一独特 “使命” 赋 予的。正是这一虚化历程使之成为元话语成分并区别于基本话语成分，同时在 这一过程中词义色彩不同的言说义动词和视觉动词 “看” 语义中和成为同类话 语标记.

基本话语发展为元话语是由语言表达互动需求促动的。Michael Hoey （2001:11）认为, 互动是语篇的中心，语篇可以定义为作者和读者之间的可见 的互动证据, 这种互动是由作者操作的有目的行为。而基本话语和元话语能够 以同一外在形式呈现源于语言自身的特点。克里斯特尔 (2000:302) 指出, 语 言的自反性（reflectiveness/reflexivity）是人类语言区别于其他符号系统的 一种特性, 指语言能用来 “谈论” 语言自身。正是语言自反性这一特质导致话 语得以区分为基本话语和元话语这两个既有区别又有联系的层面。然而正如 Crismore（1989:96）指出的那样：要想清楚地将基本话语和元话语区分开来 是不可能的，基本话语和元话语之间是非离散性的（non-discrete），很多时 候是混杂在一起的，二者构成一个连续统（Continuums）。部分总结类话语 标记兼具三种功能较好地诠释和证明基本话语和元话语之间的关系。

\section{参考文献}

Crismore, A., 1996. Talking with readers: metadiscourse as rhetorical act. New York: Peter Lang.

de Beaugrande, R. \& W. Dressler, 1981. Introduction to Text Linguistic. London: Longman.

Dooley, R. A. \& S. H. Levinson, 2008. 话语分析中的基本概念 (Analyzing Discourse: A Manual of Basic Concepts). 北京：外语教学与研 究出版社.

Grimes, J. E., 1975. The Thread of Discourse. The Hague: Mouton.

Halliday, M. A. K., 1985. An Introduction to Functional Grammar. London: Edward Arnold.

-- \& R. Hasan, 1976. Cohension in English. London: Longman.

Hoey, M., 2001. Textual Interaction: An Introduction to Written Discourse Analysis. London: Routledge.

Hopper, P. J. and E. C. Traugott, 1993. Grammaticalization. Cambridge: Cambridge University Press. 
Hyland, K., 2008, 元话语 [M]. 北京: 外语教学与研究出版社.

Langacre, R. E., 1996. The Grammar of Discourse (2th edition). New York: Plenum.

Sperber, D. \& D. Wilson, 1995. Communication and Cognition. Oxford:

Blackwell, 戴维 - 克里斯托尔, 2000, 现代语言学词典 [D]。北京: 商务印 书馆.

Verhagen, A., 2014. 交互主观性的构建：话语、句法和交际 [M]. 世界图书出 版社.

曹秀玲、辛慧, 2012 , 话语标记的多源性与非排他性 $[\mathrm{J}]$ 。语言科学，（3）： 254-262。

曹秀玲、王清华，2015, 从基本话语到元话语一一以汉语让转义 “ $X$ 然” 为例 [J]。中国语文，（6）：523-535。

李绍群, 2013, 试析总括性话语标记 “一句话” [J]。语言教学与研究, (2) : $97-10$ 。

李宇明, 拟对话语境中的 “是的” [A]。第五届国际汉语教学讨论会论文选 [C]。 北京: 北京大学出版社。

廖秋忠, 1986, 廖秋忠文集 $[\mathrm{M}]$ 。北京: 北京语言学院出版社。

刘菲露, 2008, “总之” 的语义和语用功能分析 $[J]$ 。十堰职业技术学院学报, (1) : 90-93。

刘流, 2015, 现代汉语总结类话语标记研究 [D]。上海师范大学硕士学位论文。 沈家煊, 2001, 语言的 “主观性” 和 “主观化” [J]。外语教学与研究, (4): $188-190$ 。 